\title{
FMH - Forum Notfall
}

\section{Wolfgang Ummenhofer}

Prof. em. Dr. med., SGAR-Vertreter Plattform Rettungswesen FMH, Präsident

Die Plattform Rettungswesen der FMH ist Geschichte. Und vielleicht keine ganz schlechte.

\section{Die Entwicklung des Rettungswesens in der Schweiz}

Es lohnt sich, die Thesen der FMH zum Rettungswesen in der Schweiz von 1996 anzuschauen; es ist ein kurzes Papier. Erst, wenn man realisiert, was alles für uns zwischenzeitlich zur Selbstverständlichkeit geworden ist (einheitliche Sanitätsnotrufnummer 144, ausgebildete Rettungssanitäter und Notärzte, standardisierte und zertifizierte Ausrüstung der Rettungsmittel, funktionale und kompetente Notfallstationen in unseren Spitälern), reift die Erkenntnis, dass das vor nicht allzu

\section{Die einheitliche Sanitätsnotrufnummer 144} ist zur Selbstverständlichkeit geworden.

langer Zeit eben noch nicht so war. Vermutlich war es nicht allein das Verdienst der FMH, dass sich das Rettungswesen in der Schweiz so positiv entwickelt hat aber wirkungslos waren diese Thesen sicher nicht. Zumindest entsprach es dem damaligen Selbstver- ständnis, sich nicht nur in Tariffragen, sondern auch in allgemeinen gesundheitspolitischen Fragen als Ärzteschaft zu Wort zu melden.

Jetzt ist es nicht so, dass im Rettungswesen alle Fragen gestellt und alle Probleme gelöst wären. Insbesondere der Beginn der Rettungskette ist nach wie vor eine Baustelle.

\section{Integration der ärztlichen Grundversor- genden in die Notfallbehandlung}

Aber unabhängig hiervon hat sich die Plattform vor geraumer Zeit an den Zentralvorstand gewandt und um ein kritisches Feedback zum Fortbestand des Gremiums und der weiteren Ausrichtung der Themenschwerpunkte gebeten. Die Antworten waren sehr klar: Man sei dankbar für einen interdisziplinären Think-Tank zu allen Fragen der medizinischen Notfallversorgung. Mehr denn je stehe aber die Integration der ärztlichen Grundversorgenden in die Notfallbehandlung zur Diskussion. Die kantonalen Ärztegesellschaften, verantwortlich für die Durchführung des Notfalldienstes, fühlen sich von Politik, Kostenträgern und Öffentlichkeit im Stich gelassen. Sie haben zuneh-

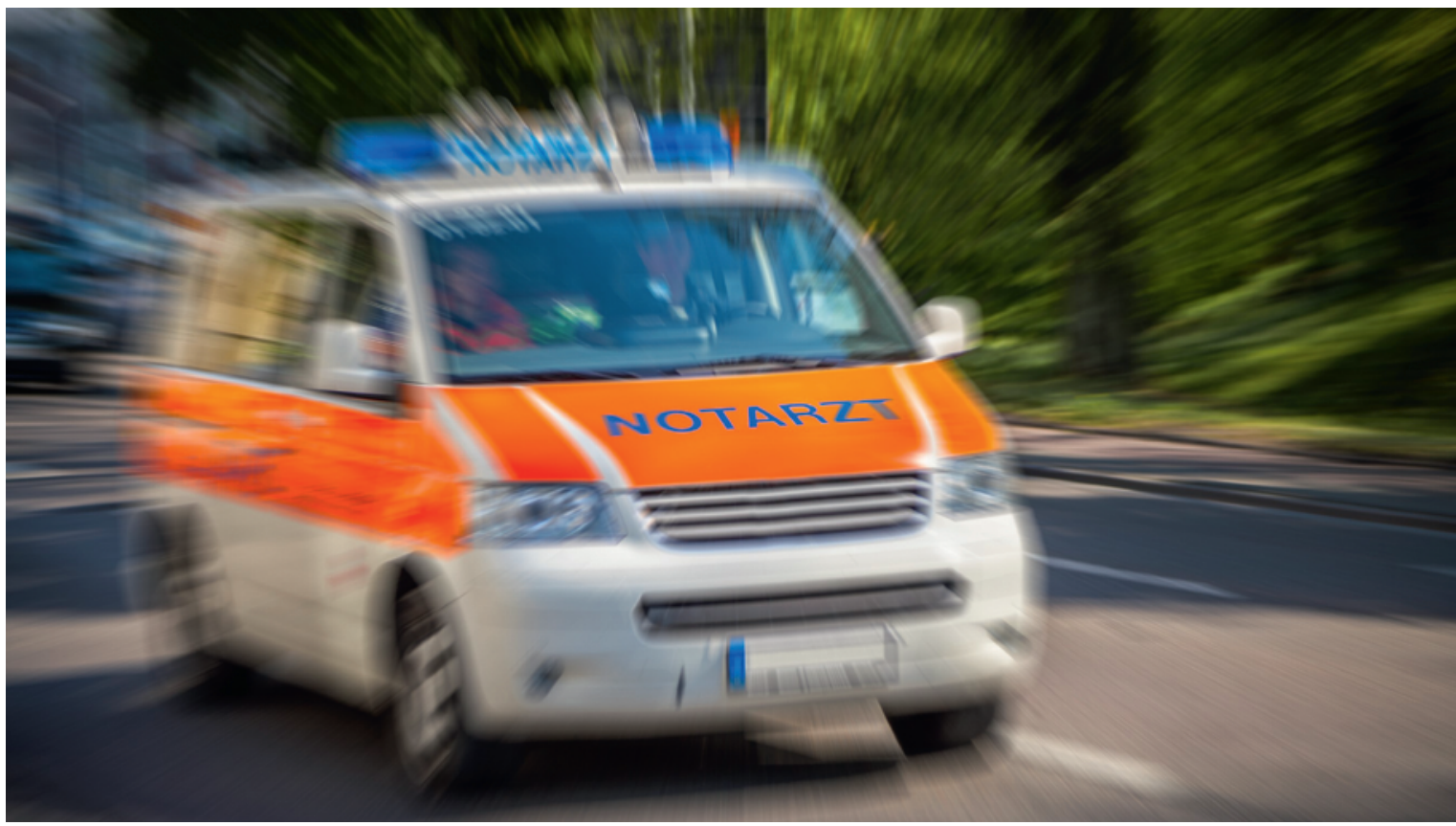


mend Schwierigkeiten, den Service sinnvoll und ressourcengerecht zu organisieren. Hausarztpraxen verwaisen, v.a. im ländlichen Raum, und nicht selten wird von potenziellen Nachfolgern die hohe Dienstbelastung bzw. der Präsenzzwang als eines der gravierenden Handicaps benannt.

\section{Verändertes Patientenverhalten}

Das alles ist kein spezifisch schweizerisches Problem und auch nicht nur mit dem (relativen) Ärztemangel zu erklären. Patienten haben ein verändertes Krankheitsverhalten; viele haben keinen Hausarzt mehr, konsultieren Triageportale oder direkt den Spezialisten und finden sich bei als Notfall empfundenen Situationen direkt auf der Notfallstation des Spitals ein.

Vielleicht liegen die Zukunftslösungen tatsächlich jenseits des klassischen Hausarztmodells. Es wäre aber hilfreich, wenn wir Ärzte uns massgeblich in die Diskussion um die strukturellen Rahmenbedingungen möglicher Zukunftsmodelle einbringen würden. Gleichgültig, wie diese aussehen, braucht es tragfähige Übergangsmodelle, die Patienten jetzt helfen und die verbleibenden Grundversorgenden vor weiterem Burn-out schützen. Der Zentralvorstand der FMH hat sich gewünscht, dass die Plattform die bisherigen Themen weiter besetzt, sich aber schwerpunktmässig mit der Rolle des Grundversorgenden im Notfallsystem beschäftigt.

Wir können dieses Bedürfnis gut nachvollziehen. Die Plattform hat sowohl in ihren Thesen [1] als auch in ihrem Leitbild 2010 zum Rettungswesen [2] die notfallmedizinische Kompetenz der grundversorgenden Dienstärzte als integrativen Baustein unseres Schwei-

\section{Namensänderungen sind immer sehr emotional.}

zer Notfallsystems hervorgehoben. Wenn sich der Fokus des Gremiums jetzt aber mehr auf die Neuorganisation der ärztlichen Notfallversorgung richtet, dann sollte sich die veränderte Orientierung auch in der Namensgebung der Gruppe niederschlagen.

Prof. em. Dr. med. Wolfgang Ummenhofer Plattform Rettungswesen FMH

Postfach 300

$\mathrm{CH}-3000$ Bern 15

w.ummenhofer[at]unibas.ch nur bei Zivilstandsangelegenheiten, auch bei uns. Nach längeren Diskussionen haben wir dem Zentralvorstand vorgeschlagen, uns in «FMH - Forum Notfall» umzubenennen, was, nach kürzerer Diskussion, jetzt einstimmig erfolgt ist. Wir wollen unsere bisherige Arbeit unter dem neuen Namen fortsetzen und freuen uns, wenn sich auch weitere Fachgesellschaften an unserer Arbeit beteiligen.

Denn dass sich in Zeiten weitgehender Fragmentierung innerhalb der Ärzteschaft 12 Fachgesellschaften und Organisationen über 25 Jahre regelmässig zum Themenkomplex medizinischer Notfallstrukturen ausgetauscht und auch zu Wort gemeldet haben, ist ja fast auch schon ein Wert an sich.

Vermutlich gibt es wenige Themengebiete in der Medizin, in denen so wenig zusammen- und so viel aneinander vorbei gearbeitet wird wie im Bereich der

\section{Wir wollen unsere bisherige Arbeit unter dem neuen Namen fortsetzen.}

medizinischen Notfallversorgung. Umso mehr, als wir Ärzte ja nur einen Teil der auf diesem Terrain engagierten «Leistungserbringer» repräsentieren und uns ja auch aus zahlreichen Funktionen mehr und mehr zurückziehen.

\section{Unterschiedliche Perspektiven offen- barten schnell die Schnittmengen}

Auf den ersten Blick waren die Synergien auch bei uns nicht immer offensichtlich. Erstversorgung, Rettungsdienst, Notfallstation, Transport kritisch kranker oder verletzter Patienten oder Krisenmanagement bei aussergewöhnlichen Lagen haben natürlich im Blickwinkel von Notfallmedizinern andere Perspektiven als bei Hausärzten, Chirurgen, Internisten, Pädiatern, Kardiologen, Psychiatern, Neurologen, Anästhesisten, Intensivmedizinern, Kantonsärzten oder dem Militär. Aber gerade aus diesen unterschiedlichen Perspektiven haben sich immer sehr schnell die Schnittmengen und die gegenseitige Vernetzung unserer Tätigkeitsfelder offenbart.

Das «FMH - Forum Notfall» würde sich freuen, wenn Sie uns wie bisher die "Plattform Rettungswesen der FMH» mit Interesse und Feedback begleiten.

Bildnachweis

(c) Huettenhoelscher | Dreamstime.com (Symbolbild)

\footnotetext{
Literatur

1 fmh.ch/files/pdf10/thesen_rettungswesen_d.pdf;

2 www.fmh.ch/files/pdf10/Leitbild1.pdf
} 